\title{
Subcarrier allocation based simultaneous wireless information and power transfer for multiuser OFDM systems
}

\author{
Zhenyu $\mathrm{Na}^{1}$, Xiaotong $\mathrm{Li}^{1}$, Xin Liü ${ }^{2^{*}}$ and Zhian Deng ${ }^{1}$
}

\begin{abstract}
Most existing algorithms of simultaneous wireless information and power transfer (SWIPT) for orthogonal frequency division multiplexing (OFDM) systems are studied based on power splitting or time splitting, which lead to time delay and the decrease of subcarrier utilization. In this paper, a multiuser OFDM system with multichannel is established and the subcarriers are divided into two parts. One part is used for information decoding and the other part is used for energy harvesting. We maximize the sum rate of the users under the constraint of energy harvesting by optimizing the channel allocation and power allocation. By means of iterative calculation, an efficient subcarrier allocation algorithm is proposed. Simulation results demonstrate that the proposed algorithm converges fast and can achieve higher sum rate than the conventional algorithm.
\end{abstract}

Keywords: Simultaneous wireless information and power transfer (SWIPT), OFDM, Subcarrier allocation, Power allocation

\section{Introduction}

Orthogonal frequency division multiplexing (OFDM) is the viable air interface for providing the high spectral efficiency and ubiquitous communication services because of its ability to combat frequency selective fading and flexibility in resource allocation. However, the powerhungry circuitries and the limited energy supplies in portable devices lead to the bottlenecks in prolonging the lifetime of networks and guaranteeing quality of service (QoS). As a result, the energy-efficient mobile communication has drawn much attention from both the industry and the academia [1-4].

Traditionally, the energy has been harvested from natural renewable energy sources, such as solar, wind and geothermal heat, thereby substantially reducing the reliance on the energy supply from conventional energy sources. In this context, simultaneous wireless information and power transfer (SWIPT) has emerged and attracted the widespread concern. Varshney put forward the concept of transmitting information and energy simultaneously and defined the capacity energy function for the first time [5]. Pulkit

\footnotetext{
* Correspondence: liuxinstar1984@dlut.edu.cn

${ }^{2}$ School of Information and Communication Engineering, Dalian University of Technology, Dalian, China

Full list of author information is available at the end of the article
}

Grover proposed the model based on the electromagnetic induction principle and analyzed the noise coupling circuit with SWIPT [6-10] maximized the energy efficiency in spectrum sensing. Two transmission protocols based on power splitting relaying and mode adaptation were proposed in OFDM relaying SWIPT systems [11]. Liang Liu proposed a classical transmission solution [12]. Specifically, the received signal is divided into two circuits: one is used for information decoding, and the other one is used for energy harvesting. As two classical models, time switching (TS) model and power switching (PS) model were put forward in [13-15], respectively. In TS model, the receiver switches to energy harvesting mode or information mode within one transmission period. In PS model, the receiver splits the power into two parts with some ratios of which one part is used for information decoding and another part is used for energy harvesting. Javer Rubio combined SWIPT with multiple-input-single-output (MISO) in [16] where a transmitter with multi-antennas transmits the same information to several banks of single antenna simultaneously. Various types of SWIPT systems including twouser MIMO broadcast channels, two-way communication links, and point-to-point links assisted by passive relays have been formulated and optimized in [17-20] proposed an optimal algorithm of power splitting based on downlink 
OFDMA by means of iterative algorithm. A tradeoff between TS and PS was proposed in [21].

As the promising technology for improving spectrum efficiency, cognitive radio (CR) has been investigated a lot recently [22-24]. SWIPT in CR networks with multiusers was studied in [25]. Two SWIPT schemes were proposed [26] for CR networks with a single SU. The secure beamforming schemes for SWIPT in a MISO broadcast channel was investigated in [27].

Different from PS and TS models, we study a subcarrier allocation algorithm based on SWIPT for the OFDM system with multiusers which is without a splitter at the receiver. The subcarriers of each user are separated into the information decoding part and the energy harvesting part. On the basis, we address the problem of maximizing the sum rate of users under the condition of enough energy harvesting. The problem is non-convex, and an iterative algorithm is used to solve it.

\section{System model}

We consider a wireless OFDM downlink system consisting of one cognitive base station (CBS) and $k$ users. As shown in Fig. 1, each user is only equipped with one antenna. Let $K$ denote the set of $k$ users. The OFDM system bandwidth is assumed to be equally divided into $n(n \geq k)$ channels. The set of subcarriers is denoted as $N(N=\{1,2, \cdots n\})$. Each subcarrier must be allocated to only one user. Parts of subcarriers are used for energy harvesting, while the others are utilized for information decoding simultaneously. We suppose that the channel power gain on each subcarrier is always constant in one transmission period provided by the

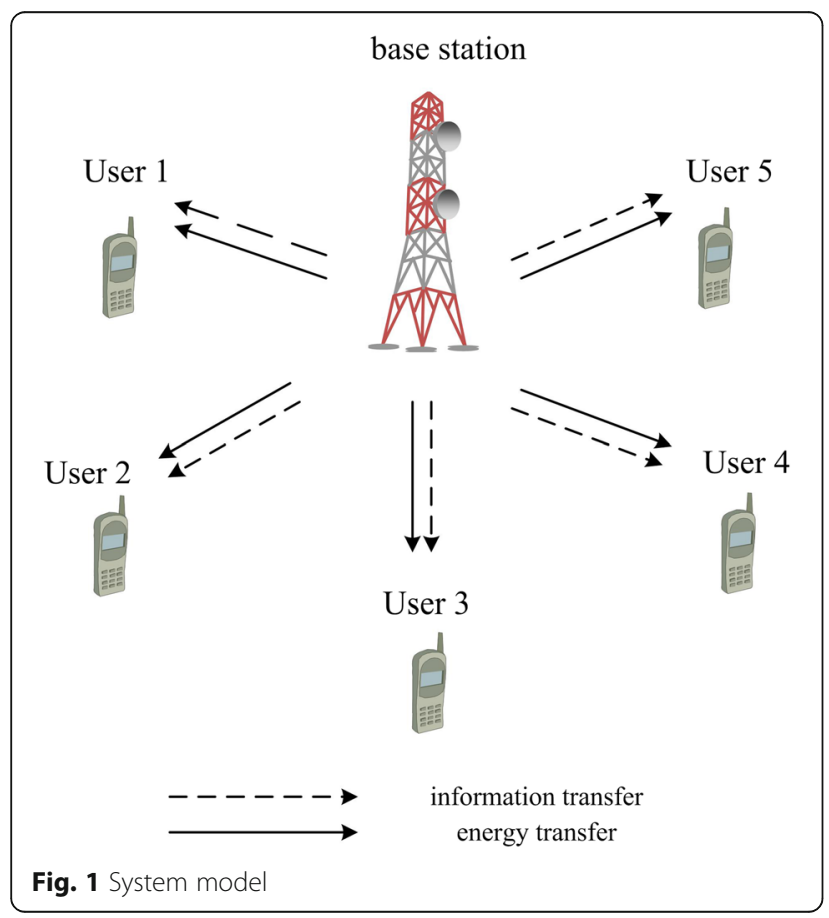

base station. Let $h_{k, n}$ represent the gain of the $k$-th user on the $n$-th subcarrier. Then, the noise power of each subcarrier is modeled as an additive white Gaussian noise (AWGN) random variable with zero mean and variance $\sigma^{2}$. The total transmission power is limited to the power budget $P$. Therefore, the power allocated on the $n$-th subcarrier is denoted as $P_{n}$. Let $S^{P}$ represent the subcarriers used for energy harvesting to power transfer. Accordingly, the other subcarriers used for information decoding are denoted by $S^{I}$. Hence, $S_{K}^{I}$ represents the subcarriers of $K$-th user for information transfer. Since one subcarrier cannot be used for energy harvesting and transfer information simultaneously, we have $S^{I} \cap S^{P}=\varnothing$ and $S^{I} \cup S^{P}=N$.

\section{Problem formulation}

Our aim is to maximize the sum rate of the OFDM downlink under the constraint of the minimum harvested energy for each user. Let $B_{k}$ represent the minimum harvested energy of the $k$-th user. Since one subcarrier can only be allocated to one user, we use $\alpha_{n, k}$ to stand for a binary channel allocation index. In other words, $\alpha_{n, k}=1$ means that the subcarrier $n$ is only allocated to the user $k$, while $\alpha_{n, k}=0$ is determined on other terms. Thus, it is written as:

$$
\sum_{k=1}^{K} \alpha_{k, n}=1, \forall n \in N
$$

The sum rate can be formulated as:

$$
\sum_{k=1}^{K} \sum_{n \in S^{I}} \alpha_{k, n} \log \left(1+\frac{h_{k, n} P_{n}}{\sigma^{2}}\right)
$$

Here, $n \in S^{I}$. With energy harvesting efficiency $\varepsilon$, the harvested energy during one transmission block for user $k$ is determined by:

$$
\sum_{n \in S^{P}}\left(\varepsilon h_{k, n} P_{n}+\sigma^{2}\right)
$$

For $\forall k \in K$. Therefore, the optimization model of maximum sum rate can be expressed as:

$$
\begin{aligned}
& \max _{\alpha_{n, k}, S^{I}, P_{n} \sum_{k=1}^{K} \sum_{n \in S^{I}} \alpha_{k, n} \log \left(1+\frac{h_{k, n} P_{n}}{\sigma^{2}}\right)} \\
& \quad \text { s.t. } \sum_{n \in N} P_{n} \leq P \quad\left(P_{n} \geq 0\right) \\
& S^{P} \cup S^{I}=N \\
& S^{P} \cap S^{I}=\varnothing \\
& \sum_{k=1}^{K} \alpha_{k, n}=1, \quad \forall n \in N \\
& \alpha_{k, n} \in\{0,1\}, \quad \forall k \in K, n \in N
\end{aligned}
$$

\section{Optimal solution}

Since the problem is non-convex, it is impossible to obtain the optimal solution directly. In this section, a sub-optimal algorithm is proposed for solving the non-convex problem. 
The optimization task can be decomposed into three steps: to optimize $\alpha_{k, n}$ with given $P_{n}$ and $S^{I}\left(S^{P}\right)$, to optimize $P_{n}$ with given $\alpha_{k, n}$ and $S^{I}\left(S^{P}\right)$, as well as to optimize $S^{I}\left(S^{P}\right)$ with given $\alpha_{k, n}$ and $P_{n}$.

Firstly, since $P_{n}$ and $S^{I}\left(S^{P}\right)$ are determined, $\alpha_{k, n}$ is optimized as follows:

$$
\begin{array}{r}
\max _{n, k} \sum_{k=1}^{K} \alpha_{k, n} \log \left(1+\frac{h_{k, n} P_{n}}{\sigma^{2}}\right), \quad n \in S^{I} \\
\text { s.t. } \sum_{k=1}^{K} \alpha_{k, n}=1, \quad \forall n \in N \\
\alpha_{k, n} \in\{0,1\}, \quad \forall k \in K, n \in N
\end{array}
$$

The problem above is regarded as allocating the subcarrier $n$ to the assigned user for obtaining the maximum sum rate. In other words, the subcarrier $n\left(n \in S^{I}\right)$ is allocated to the user $k$ which can get the maximum $h_{k, n} P_{n}$, i.e., $\alpha_{k^{*}, n}=1, k^{*}=\arg \max _{k \in K} h_{k, n} P_{n}$ and $\alpha_{k, n}=$ $0, \forall k \neq k^{*}, k \in K$.

Secondly, $P_{n}$ is optimized by $\alpha_{k, n}$ and $S^{I}\left(S^{P}\right)$. In this proposition, the problem can be rewritten as:

$$
\begin{array}{cl}
\max _{P_{n}} & \sum_{n \in S_{k}^{I}} \log \left(1+\frac{h_{k^{*}, n} P_{n}}{\sigma^{2}}\right) \\
\text { S.t. } & \sum_{n \in S^{P}}\left(\varepsilon h_{k^{*}, n} P_{n}+\sigma^{2}\right) \geq B_{k} \\
& \sum_{n \in N} P_{n} \leq P \quad\left(P_{n} \geq 0\right)
\end{array}
$$

Note that $\alpha_{k^{*}, n}=1, \alpha_{k, n}=0, \forall k \neq k^{*}, k \in K$. The converted problem is satisfied with convex model. Therefore, the Lagrange dual decomposition is adopted to solve this problem. The Lagrange dual function is as follows:

$$
g\left(\beta_{1}, \beta_{2}\right)=\max _{\left\{P_{n}\right\}} L\left(P_{n}\right)
$$

Where, $\beta_{1}$ and $\beta_{2}$ are the Lagrange multipliers and they are determined by the sub-gradient method. Meanwhile, $L\left(P_{n}\right)$ is expressed as:

$$
\begin{aligned}
L\left(P_{n}\right)= & \sum_{n \in S_{k}^{l}} \log \left(1+\frac{h_{k^{*}, n} P_{n}}{\sigma^{2}}\right) \\
& +\beta_{1}\left\{\sum_{n \in S^{P}}\left(\varepsilon h_{k^{*}, n} P_{n}+\sigma^{2}\right)-B_{k}\right\} \\
& +\beta_{2}\left(P-\sum_{n \in N} P_{n}\right)
\end{aligned}
$$

Then, the dual problem can be simplified as follows:

$$
\begin{aligned}
& \min _{\beta_{1}, \beta_{2}} g\left(\beta_{1},, \beta_{2}\right) \\
& \text { s.t } \quad \beta_{1}, \beta_{2} \geq 0
\end{aligned}
$$

Because the dual problem is differentiable, it can be solved by the classic sub-gradient method, which solves the optimal problem based on the gradient and the suitable step size [28]. The result is presented as below:

$$
\begin{aligned}
& \Delta \beta_{1}=\sum_{n \in S^{D}} \varepsilon h_{k^{*}, n} P_{n}+\sigma^{2}-B_{k} \\
& \Delta \beta_{2}=P-\sum_{n \in N} P_{n}
\end{aligned}
$$

For the given $\beta_{1}$ and $\beta_{2}$, the optimal power $P_{n}\left(n \in S^{I}\right)$ is obtained according to KKT conditions using mathematical manipulation:

$$
P_{n}=\left(\frac{1}{\beta_{2}}-\frac{\sigma^{2}}{h_{k^{*}, n}}\right)^{+}
$$

Where, (.) denotes $\max (., 0)$.

Similarly, the allocated power $P_{n}$ used for energy harvesting is determined as:

$$
P_{n}=\left\{\begin{array}{l}
P_{\max } \beta_{1} h_{k^{*}, n} \varepsilon>\beta_{2} \\
P_{\min } \beta_{1} h_{k^{*}, n} \varepsilon \leq \beta_{2}
\end{array}\right.
$$

Where, $P_{\max }$ and $P_{\min }$ represent the maximum and minimum power constraints on information decoding, respectively.

\section{Algorithm 1: Proposed algorithm for the problem (6)}

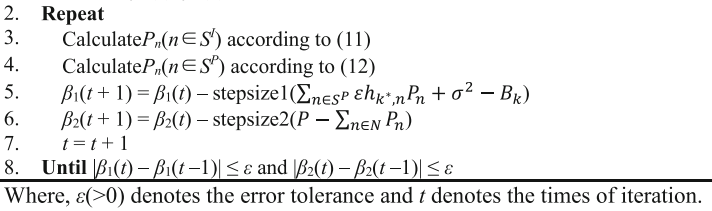

According to $P_{n}$ and $\alpha_{n, k}, S^{I}\left(S^{P}\right)$ can be obtained by substituting (11) and (12) into (8). Consequently, the Lagrange dual function can be rewritten as below:

$$
\begin{aligned}
L\left(S^{P}\right)= & \sum_{k=1}{ }^{K} \sum_{n \in N} \alpha_{n, k} \log \left(1+\frac{h_{k, n} P_{n}}{\sigma^{2}}\right) \\
& -\sum_{k=1} \sum^{K} \sum_{n \in S^{p}} \alpha_{n, k} \log \left(1+\frac{h_{k, n} P_{n}}{\sigma^{2}}\right) \\
& +\beta_{1} \sum_{n \in S^{p}}\left(\varepsilon h_{k, n} P_{n}+\sigma^{2}\right)-\beta_{1} B_{k}+\beta_{2} P-\beta_{2} \sum_{n \in N} P_{n} \\
= & \sum_{n \in S^{p}}\left\{\beta_{1}\left(\varepsilon h_{k, n} P_{n}+\sigma^{2}\right)-\sum_{k=1}^{K} \alpha_{n, k} \log \left(1+\frac{h_{k, n} P_{n}}{\sigma^{2}}\right)\right\} \\
& +\sum_{n \in N}\left\{\sum_{k=1}^{K} \alpha_{n, k} \log \left(1+\frac{h_{k, n} P_{n}}{\sigma^{2}}\right)-\beta_{2} P_{n}\right\}-\beta_{1} B_{k}+\beta_{2} P \\
=\sum_{n \in S^{p}} F_{n} & +\sum_{n \in N}\left\{\sum_{k=1}^{K} \alpha_{n, k} \log \left(1+\frac{h_{k, n} P_{n}}{\sigma^{2}}\right)-\beta_{2} P_{n}\right\}-\beta_{1} B_{k}+\beta_{2} P
\end{aligned}
$$

Where, 


$$
F_{n}=\beta_{1}\left(\varepsilon h_{k, n} P_{n}+\sigma^{2}\right)-\sum_{k=1}^{K} \alpha_{n, k} \log \left(1+\frac{h_{k, n} P_{n}}{\sigma^{2}}\right)
$$

When we observe the formula (13), only the first item on the right side is about to $S^{P}$. Thus, the optimal $S^{P}$ can be achieved by maximizing the item $F_{n}$, i.e.,

$$
S^{P *}=\arg \max _{S^{P}} \sum_{n \in S^{P}} F_{n}^{*}
$$

$S^{P_{*}}$ can be easily obtained by substituting all the $n$ into $F_{n}$ to find the ones which make $F_{n}$ positive. As a result, the rest of the set $N$ belongs to $S^{I n}$.

The proposed algorithm to solve the optimal problem is presented as below:

Algorithm 2: Proposed algorithm for the joint optimizationproblem

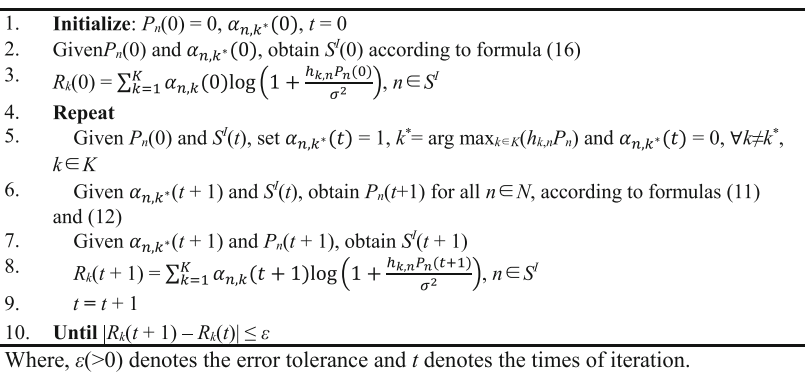

\section{Simulation results}

In this section, the performance of the proposed subcarrier allocation algorithm based on SWIPT for the OFDM system with multiusers is demonstrated by simulation results.

We denote all the channels involved follow the Rayleigh distribution. For simplicity, we suppose that the minimum harvested energy limits for all the users are

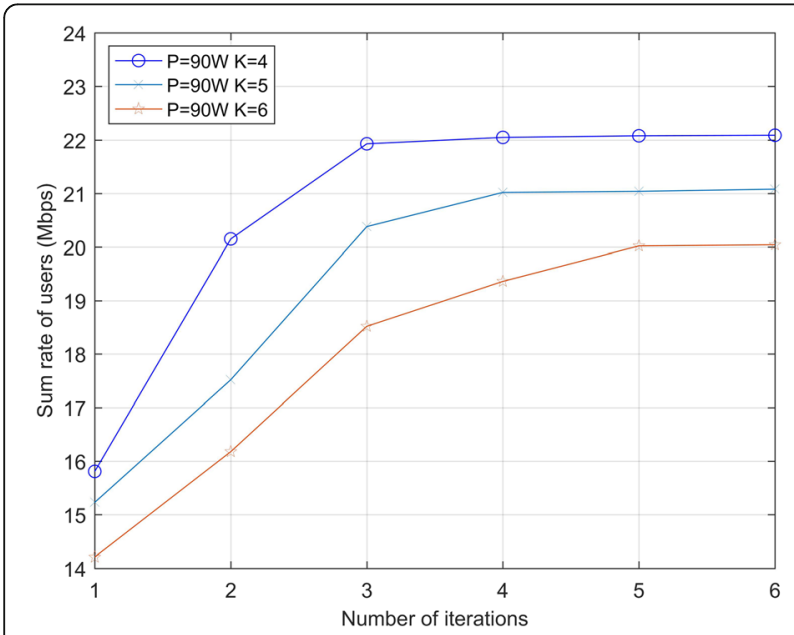

Fig. 2 Convergence behavior of the proposed algorithm

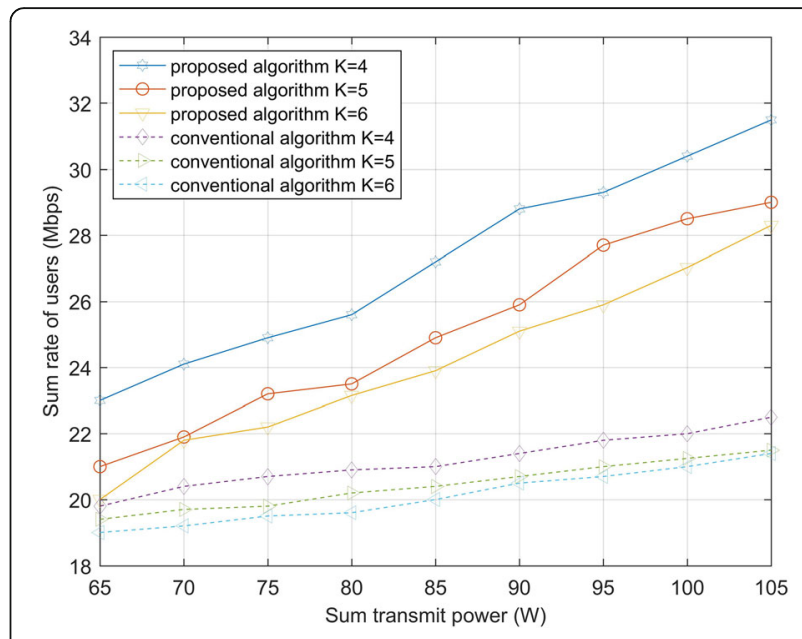

Fig. 3 Sum rate of users vs. sum transmission power

the same, i.e., $B_{k}=B$. In addition, we set $N=16, K=5$, $\sigma^{2}=1, P_{\max }=3 \mathrm{~W}, P_{\min }=0, \varepsilon=1$, and the bandwidth is equal to $1 \mathrm{MHz}$.

Figure 2 shows the convergence behavior of the proposed algorithm. It can be seen that the proposed algorithm converges fast. It indicates that the proposed algorithm can be implemented practically.

Figure 3 presents the comparison between the proposed optimization algorithm and the conventional algorithm of subcarrier allocation based on [29]. It can be observed that the proposed algorithm performs better compared with the conventional algorithm.

When the conventional algorithm allocates $N$ subcarriers to $K$ users, all the subcarriers are used for information decoding and the consumed energy comes from the system. Since the system can not produce energy by itself, the energy comes from the finite battery. Therefore, the constraint of minimum energy harvesting can not be

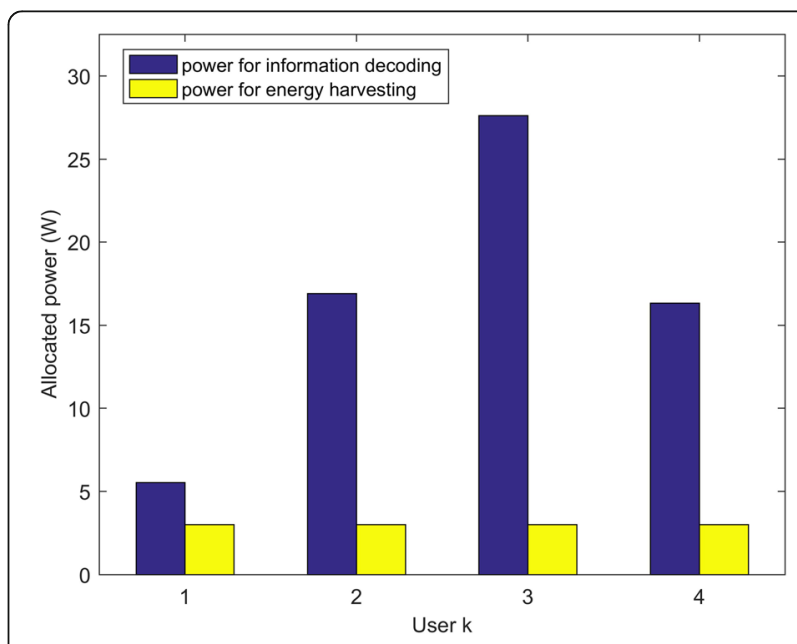

Fig. 4 The allocated power for user $k$ 


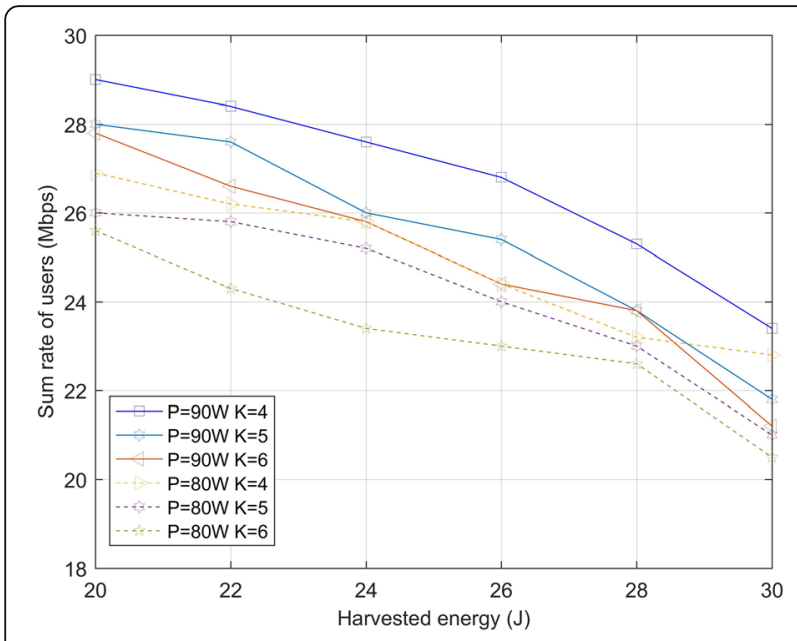

Fig. 5 Sum rate of users vs. harvested energy

satisfied more often than not. Since the water-filling approach is used for the power allocation of the conventional algorithm, it would cause some power waste. What is more, all the subcarriers allocated to information decoding lead to the energy consumption and less power is used for information decoding. Figure 2 also demonstrates that the sum rate of users increases with the increase of sum transmit power $P$. It can be interpreted as that the increase of sum transmit power brings about the more power allocated to information decoding with the same target harvested energy.

Figure 4 shows that the total transmit power used for information decoding of user $k$. It can be seen that the user 3 is allocated the most power, while the user 1 is the least. This is because, in our simulation, the user 3 has the best channel condition so that it can achieve higher sum rate.

Figure 5 shows that the sum rate of users versus the minimum energy harvesting limit $B_{k}$ under the fixed power budget. It is obvious that the sum rate varies with the constraint of the harvested energy. When $B_{k}$ increases, the sum rate decreases. This is because when the constraint of energy harvesting increases, more subcarriers are allocated to satisfy the constraint of the harvested energy. It leads to less resources that are allocated to the information decoding. It can also be observed that, with regard to the same constraint of energy harvesting, the sum rate decreases as the number of users increases. That is expected due to the fact that under the same power budget, the increasing number of users causes the decrease of the average allocated power for each subcarrier. As a result, more subcarriers are allocated to energy harvesting in order to satisfy the same constraint of the required energy, as well as the sum rate of users increases.

Figure 6 shows the allocated power to each subcarrier under the condition of the power budget equal to $80 \mathrm{~W}$. It also illustrates the average channel gain for each subcarrier. As shown in Fig. 6, when the subcarrier has the good channel condition, it is always used for energy harvesting. On the other hand, when the subcarrier has the bad channel condition, it is always used for information decoding and less power is allocated. The phenomenon appears because to allocate the good channel for energy harvesting leads to less subcarriers used for energy harvesting and more subcarriers used for information decoding to achieve maximum sum rate.
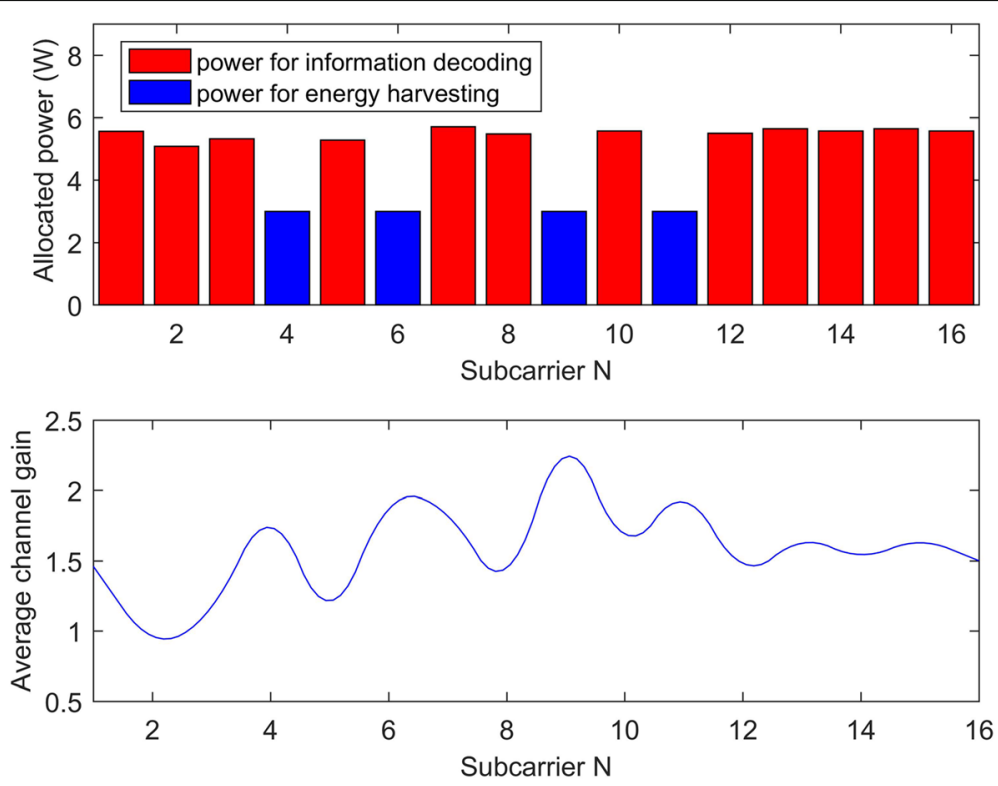

Fig. 6 The allocated power and channel condition of subcarrier $n$ 


\section{Conclusions}

A subcarrier allocation algorithm based on SWIPT for the OFDM system with multiusers was proposed in this paper. Traditionally, SWIPT is used for single user in OFDM systems more often than not. However, we proposed a joint optimization algorithm used for multiusers. Specifically, the OFDM subcarriers of each user are divided into two parts whose one part is used for information decoding and the other part is used for energy harvesting. Therefore, in contrast to the conventional time or power splitter at the receiver, the enough information rate can be obtained on the premise of the harvested energy we require. Simulation results show that the proposed algorithm converges fast and performs better than the conventional algorithm in the rate of information decoding.

\section{Funding}

This work was supported by the National Natural Science Foundations of China under Grant No. 61301131 and 61601221, the Natural Science Foundations of Jiangsu Province under Grant No. BK20140828, the China Postdoctoral Science Foundations under Grant No. 2015M580425 and the Fundamental Research Funds for the Central Universities under Grant No. 3132016347 and DUT16RC(3)045.

\section{Authors' contributions}

ZN designed the main body of the algorithm and completed the first simulation. $X L$ gave the guidance on the ideas of the paper, optimized the parameters, and discussed the related problems with other authors. $\mathrm{XL}$ completed the remaining simulations, analyzed data, and processed simulation results. All authors read and approved the final manuscript.

\section{Authors' information \\ ${ }^{1}$ Zhenyu $\mathrm{Na}$ and Xiaotong Li are from the School of Information Science and Technology of Dalian Maritime University (DMU), Dalian, China. Zhenyu Na is the associate professor of DMU. His research interests include OFDM communications, non-orthogonal multicarrier techniques, satellite communications, and networking. Xiaotong $\mathrm{Li}$ is the postgraduate of DMU whose research field is the OFDM communications and systems, information and energy transfer optimization in wireless multicarrier systems. \\ ${ }^{2}$ Xin Liu is the associate professor of the School of Information and Communication Engineering of Dalian University of Technology (DUT), Dalian, China. His research interests include cognitive radio, SWIPT, joint resource management in mobile communication systems, satellite communications, and space laser communications.}

\section{Competing interests}

The authors declare that they have no competing interests.

\section{Publisher's Note}

Springer Nature remains neutral with regard to jurisdictional claims in published maps and institutional affiliations.

\section{Author details}

${ }^{1}$ School of Information Science and Technology, Dalian Maritime University, Dalian, China. ${ }^{2}$ School of Information and Communication Engineering, Dalian University of Technology, Dalian, China.
Received: 17 July 2017 Accepted: 17 August 2017

Published online: 02 September 2017

\section{References}

1. T. Chen, Y. Yang, H. Zhang, H. Kim, K. Horneman, Network energy saving technologies for green wireless access networks. IEEE Wirel. Commun. 18(5), 30-38 (2011)

2. F. Zhao, X. Sun, H. Chen, R. Bie, Outage performance of relay-assisted primary and secondary transmissions in cognitive relay networks. EURASIP J. Wirel. Commun. Netw. 2014, 60(1) (2014)

3. M. Jia, L. Wang, Q. Guo, A low complexity detection algorithm for fixed up-link SCMA system in mission critical scenario. IEEE Int Things J 1(1), 99 (2017)

4. M. Jia, Z. Gao, Q. Guo, Fast compressive tracking with robust example selection based on multiple instance learning in smart and autonomous systems. Pattern Recognition. 69, 325-335(2017)

5. L.R. Varshney, Transporting Information and Energy Simultaneously. IEEE International Symposium on Information Theory. IEEE (2016), pp. 1612-1616

6. P. Grover, A. Sahai, Shannon meets Tesla: wireless information and power transfer (2010), pp. 2363-2367

7. J. Guo, N. Zhao, F.R. Yu, X. Liu, Exploiting adversarial jamming signals for energy harvesting in interference networks. IEEE Trans. Wirel. Commun. 16(2), 1267-1280 (2017)

8. W. Zhong, K. Chen, X. Liu, Joint optimal energy-efficient cooperative spectrum sensing and transmission in cognitive radio. IEEE Wirel. Commun. 14(1), 98-110 (2014)

9. M. Jia, X. Liu, X. Gu, Joint cooperative spectrum sensing and channel selection optimization for satellite communication systems based on cognitive radio. Int. J. Satell. Commun. Netw. 23(3), 139-150(2015)

10. M. Jia, X. Gu, Q. Guo, Broadband hybrid satellite-terrestrial communication systems based on cognitive radio toward 5G. IEEE Wirel. Commun. 23(6), 96-106 (2013)

11. Y. Liu, X. Wang, Information and energy cooperation in OFDM relaying: Protocols and optimization. IEEE Trans. Veh. Technol. 65(7), 5088-5098 (2016)

12. L. Liu, R. Zhang, K.C. Chua, Secrecy wireless information and power transfer with MISO beamforming. IEEE Trans. Signal Process. 62(7), 1850-1863 (2014)

13. L. Liu, R. Zhang, K.C. Chua, Wireless information transfer with opportunistic energy harvesting. IEEE Trans. Wirel. Commun. 12(1), 288-300 (2012)

14. F. Zhao, L. Wei, H. Chen, Optimal time allocation for wireless information and power transfer in wireless powered communication systems. IEEE Trans. Veh. Technol. 65(3), 1830-1835 (2016)

15. L. Liu, R. Zhang, C.K. Ho, Wireless information and power transfer: a dynamic power splitting approach. IEEE Trans. Wirel. Commun. 61(9), 3990-4001 (2013)

16. D.W.K. Ng, R. Schober, Spectral efficient optimization in OFDM systems with wireless information and power transfer. IEEE Wirel. Commun. 6(2), 1-5 (2013)

17. R. Zhang, C. Ho, MIMO broadcasting for simultaneous wireless information and power transfer. IEEE Trans. Wirel. Commun. 12(5), 1989-2001 (2013)

18. F. Zhao, B. Li, H. Chen, X. LV, Joint beamforming and power allocation for cognitive MIMO systems under imperfect CSI based on game theory. Wirel. Pers. Commun. 73(3), 679-694 (2013)

19. F. Zhao, W. Wang, H. Chen, Q. Zhang, Interference alignment and gametheoretic power allocation in MIMO heterogeneous sensor networks communications. Signal Process. 126(C), 173-179 (2016)

20. X. Zhou, R. Zhang, C.K. Ho, Wireless information and power transfer in multiuser OFDM systems. IEEE Trans. Wirel. Commun. 13(4), 2282-2294 (2014)

21. D.W.K. Ng, E.S. Lo, R. Schober, Wireless information and power transfer: energy efficiency optimization in OFDMA systems. IEEE Trans. Wirel. Commun. 12(12), 6352-6370 (2013)

22. X. Liu, M. Jia, X.Z. Tan, Threshold optimization of cooperative spectrum sensing in cognitive radio network. Radio Sci. 48(1), 23-32 (2013)

23. X. Liu, G.A. Bi, M. Jia, Y.L. Guan, Joint optimization of sensing threshold and transmission power in wideband cognitive radio. Radio Sci. 48(4), 359-370 (2013)

24. F. Zhao, H. Nie, H. Chen, Group buying spectrum auction algorithm for fractional frequency reuses cognitive cellular systems. Ad Hoc Netw. 58, 239-246 (2017)

25. L. Sibomana, H.J. Zepernick, H. Tran, Signal Processing and Communications Systems (ICSPCS), 2014 8th international conference on, Wireless information and power transfer in an underlay cognitive radio network (2014), pp. 1-7

26. Z. Gao, B. Wang, Wireless Communication \& Signal Processing (WCSP) 2015 International Conference on. User cooperation in OFDM-based cognitive radio networks with simultaneous wireless information and power transfer (2015), pp. 1-6 
27. D.W.K. Ng, R. Schober, 2003 IEEE GlobeconWorkshops (GC Wkshps), Resource allocation for secure communication in systems with wireless information and power transfer (2013), pp. 1251-1257

28. A. Nedic, A. Ozdaglar, Distributed subgradient methods for multi-agent optimization. IEEE Trans. Autom. Control 54(1), 48-61 (2007)

29. W. Lu, Y. Gong, J. Wu, Simultaneous wireless information and power transfer based on joint subcarrier and power allocation in OFDM systems. IEEE Access 1(1), 99 (2017)

\section{Submit your manuscript to a SpringerOpen ${ }^{\bullet}$ journal and benefit from:}

- Convenient online submission

- Rigorous peer review

- Open access: articles freely available online

- High visibility within the field

- Retaining the copyright to your article

Submit your next manuscript at $\gg$ springeropen.com 